\title{
Familiarity with a vocal category biases the compartmental expression of Arc/Arg3.1 in core auditory cortex
}

\author{
Tamara N. Ivanova, ${ }^{1}$ Christina Gross, ${ }^{2,3,5}$ Rudolph C. Mappus, ${ }^{1}$ Yong Jun Kwon, ${ }^{1,4}$ \\ Gary J. Bassell, ${ }^{2,3}$ and Robert C. Liu ${ }^{1,3}$ \\ ${ }^{1}$ Department of Biology, Emory University, Atlanta, Georgia 30322, USA; ${ }^{2}$ Department of Cell Biology, Emory University School of \\ Medicine, Atlanta, Georgia 30322, USA; ${ }^{3}$ Center for Translational Social Neuroscience, Emory University, Atlanta, Georgia 30322, USA; \\ ${ }^{4}$ Graduate Program in Neuroscience, Laney Graduate School, Emory University, Atlanta, Georgia 30322, USA
}

\begin{abstract}
Learning to recognize a stimulus category requires experience with its many natural variations. However, the mechanisms that allow a category's sensorineural representation to be updated after experiencing new exemplars are not well understood, particularly at the molecular level. Here we investigate how a natural vocal category induces expression in the auditory system of a key synaptic plasticity effector immediate early gene, Arc/Arg3.1, which is required for memory consolidation. We use the ultrasonic communication system between mouse pups and adult females to study whether prior familiarity with pup vocalizations alters how Arc is engaged in the core auditory cortex after playback of novel exemplars from the pup vocal category. A computerized, 3D surface-assisted cellular compartmental analysis, validated against manual cell counts, demonstrates significant changes in the recruitment of neurons expressing Arc in pup-experienced animals (mothers and virgin females "cocaring" for pups) compared with pup-inexperienced animals (pup-naïve virgins), especially when listening to more familiar, natural calls compared to less familiar but similarly recognized tonal model calls. Our data support the hypothesis that the kinetics of Arc induction to refine cortical representations of sensory categories is sensitive to the familiarity of the sensory experience.
\end{abstract}

The ability to recognize novel exemplars of behaviorally relevant sensory categories is critical to navigating our environment and communicating with others. A sensory system must presumably assess whether a stimulus matches the collection of features that statistically define a recognized category. At the same time, variation present in new exemplars must themselves be incorporated into the sensory system's representation of that category. The neural mechanisms that underlie such categorization at the electrophysiological level are being elucidated in both the visual and auditory modalities (DiCarlo et al. 2012; Bizley and Cohen 2013; Shepard et al. 2015), but the molecular mechanisms that support the learning and memory of sensory categories remain poorly understood.

One promising molecular coordinator of activity-dependent changes in synaptic efficacy, which has been suggested to underlie learning and memory, is the effector immediate early gene (IEG) Arc/Arg3.1 (Arc), a "master regulator" of synaptic plasticity during information processing (Shepherd and Bear 2011). Arc is expressed in hippocampal neurons that then undergo synaptic plasticity as a result of a behavioral experience (Guzowski et al. 1999; Vazdarjanova and Guzowski 2004; Fletcher et al. 2006). It can also be induced in sensory systems after sensory experiences (Tagawa et al. 2005; Carpenter-Hyland et al. 2010; Ivanova et al. 2011; Morin et al. 2011). Moreover, the levels of Arc expression correlate with hippocampus-dependent learning (Guzowski et al. 2001), plays es-

\footnotetext{
5Present address: Division of Neurology, Cincinnati Children's Hospital Medical Center, Cincinnati, OH 45229, USA.

Corresponding author: robert.liu@emory.edu

Article is online at http://www.learnmem.org/cgi/doi/10.1101//m.046086. 117.
}

sential roles in homeostatic plasticity and the long-term consolidation of memories (Gao et al. 2010). Recent studies suggest this may stem from its apparent preferential "inverse tagging" in activated neurons of the subset of inactive synapses, which then undergo long-term depression by endocytosis of AMPA receptors (Waung et al. 2008; Okuno et al. 2012). Arc's action explains how repeated experiences with the same visual stimulus leads to a progressively smaller population of visual cortical neurons that is more robustly activated by that stimulus (Wang et al. 2006), thereby helping to refine the stimulus specificity of sensory neurons. In the real world though, the exact same experience or stimulus is not usually encountered. Instead, natural stimuli are more likely to fall into the same behaviorally relevant stimulus category, but differ in their physical detail from exemplar to exemplar. Given Arc's role in refining synaptic connectivity for a neuron's stimulus specificity, how might it be deployed to support plasticity for stimulus generalization to novel exemplars of a familiar stimulus category?

We address this question in the context of natural auditory categorization of behaviorally relevant communication sounds in a mouse model. Maternal females recognize the ultrasonic vocalizations (USV) of displaced mouse pups as behaviorally important, and will seek out and retrieve the lost pup to the nest. On the other hand, nonmaternal, virgin females do not show a behavioral preference for pup calls over neutral sounds, until they have had

(C) 2017 Ivanova et al. This article is distributed exclusively by Cold Spring Harbor Laboratory Press for the first 12 months after the full-issue publication date (see http://learnmem.cshlp.org/site/misc/terms.xhtml). After 12 months, it is available under a Creative Commons License (Attribution-NonCommercial 4.0 International), as described at http://creativecommons.org/licenses/by-nc/ $4.0 /$. 
experience "cocaring" for pups—suggesting that the behavioral relevance of pup USVs is acquired (Ehret et al. 1987; Lin et al. 2013). These whistle-like calls are naturally variable along various acoustic dimensions, such as frequency, duration, frequency modulation, and bandwidth (Liu et al. 2003; Grimsley et al. 2011), but nevertheless form a distinct acoustic category that can be categorically perceived by maternal mice (Ehret and Haack 1981; Ehret 1992). Importantly, excitatory plasticity in a subset of putative pyramidal neurons in the core auditory cortex of maternal females enhances the spiking response to favor the combination of acoustic features that are statistically predictive of the pup USV category, an effect not observed in nonmaternal virgins (Shepard et al. 2015). Hence, for maternal but not nonmaternal mice, pup USVs form a familiar, behaviorally relevant sound category that core auditory cortical activity "learns" to systematically differentiate from other sound categories.

The mounting electrophysiological evidence for plasticity in auditory cortex in this vocalization recognition paradigm provides a basis for studying the molecular mechanisms involved (Liu and Schreiner 2007; Galindo-Leon et al. 2009; Cohen et al. 2011; Lin et al. 2013; Marlin et al. 2015). In particular, it opens a unique opportunity to investigate in a natural context how Arc is expressed in auditory cortex when new sounds (models of pup USVs) either do (maternal mice) or do not (nonmaternal mice) conform to a familiar, behaviorally relevant category. We assessed this using a cell compartmental analysis of Arc gene transcription by fluorescent in situ hybridization and 3D surface reconstruction (Guzowski et al. 1999; Ivanova et al. 2011). We have previously used these methods to demonstrate that prior history with a pure tone can shift how that tone induces compartmental $A r c$ expression in core auditory cortical neurons, such that, soon after stimulation, Arc is found more often in just the perinuclear cytoplasmic (perinuc) compart- ment in mice that previously heard the exact same tone compared with those that had not.

To further test and extend our interpretation of this stimulus "familiarity" effect in compartmental Arc expression, we now ask in a completely different auditory paradigm-the maternal pup USV recognition model-whether the same bias for perinuc-only expression can be induced by novel exemplars containing acoustic features congruent with a natural sound category that is either familiar or not familiar to the animal. Our data suggest that the more similar the combination of acoustic features in the exemplars is to a familiar, behaviorally relevant category, the earlier a bias for perinuc-only Arc expression is observed in core auditory cortical neurons.

\section{Results}

In the present study, we used the maternal recognition paradigm (Fig. 1A,B) to investigate how familiarity with sounds (Fig. 1C) can change compartmentalization of Arc mRNA (Fig. 2) in auditory cortical neurons. The dentate gyrus was used as a positive control area for confocal microscopy (Guzowski et al. 1999; Vazdarjanova et al. 2002; Vazdarjanova and Guzowski 2004), in which a small number of Arc mRNA strongly labeled cells can always be detected (Fig. 2A). Here and in our previous study (Ivanova et al. 2011) we used computer-generated 3D surface reconstruction of nuclei and Arc mRNA (Fig. 2B) by objectively thresholding fluorescence in the confocal $z$-stacks.

To validate our calculations against the typical approaches taken in the literature, we compared our 3D surface counting results (Fig. 3, Ims1) to manual counting by methods previously described by Chawla et al. (2004). For such manual counting, 3D

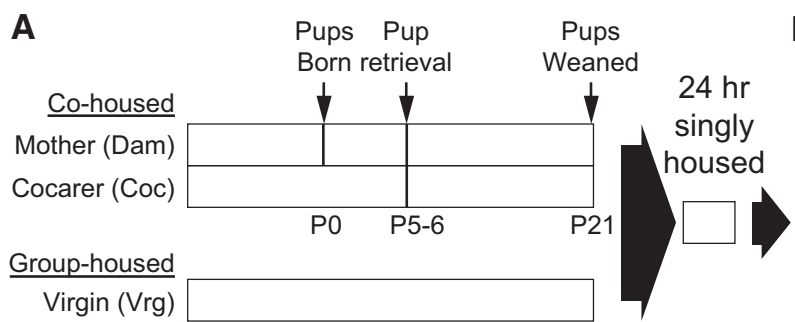

C

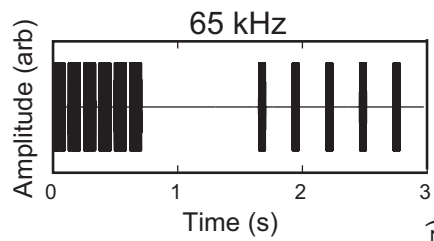

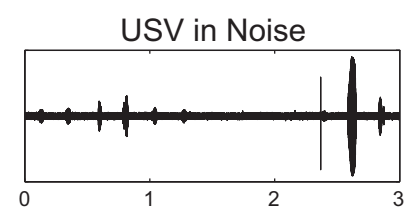

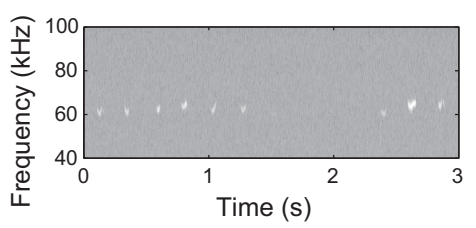

B
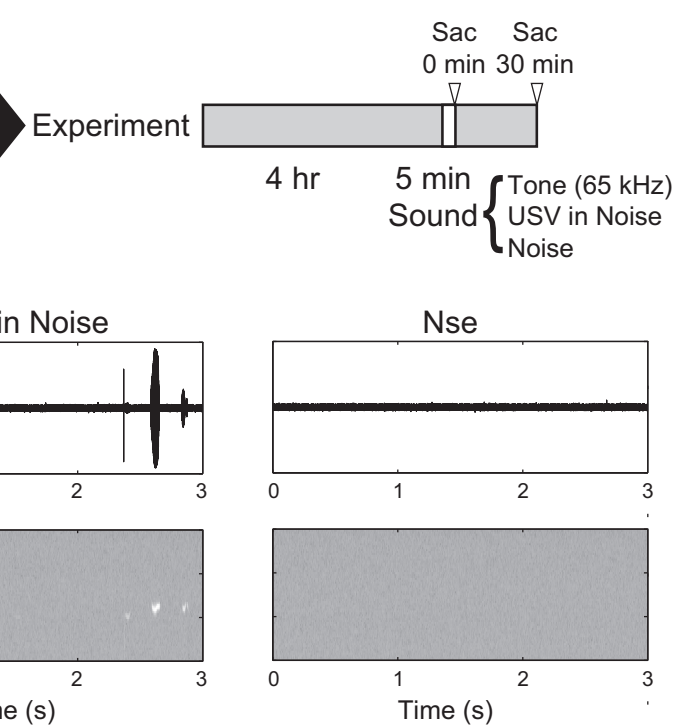

Figure 1. Experimental paradigm. (A) Preexperiment timeline for each of three groups of adult female mice. Dams and Cocs were cohoused for several days before pups were born, and then screened on P5 or P6 for successful retrieval of scattered, vocalizing pups in the home cage. Pups were weaned on P21, after which Dams and Cocs were separated into individual cages for $24 \mathrm{~h}$. Vrgs, matched to Dams and Cocs, had no adult contact with pups, and were group housed in the colony over the same period of time before being placed into an individual cage $24 \mathrm{~h}$ prior the experiment. (B) Timeline for sound exposure on experiment day. An individual mouse in its home cage was placed into a silent anechoic chamber for a 4-h habituation in silence, followed by a 5 -min period of sound stimulation by Tone (65 kHz), USV in Noise, or Noise. Mice were decapitated immediately afterwards (Sac 0 min) or with a 30-min delay (Sac $30 \mathrm{~min}$ ). (C) (Left) Waveform of the first $3 \mathrm{sec}$ of the random sequence of pure tone pips at $65 \mathrm{kHz}$, a synthetic model of pup USVs. (Center) Waveform (top) and spectrograms (bottom) of the first $3 \mathrm{sec}$ of the USV and the matched Noise (Right) stimulus. Gray scale indicates higher amplitude with whiter pixels. 
A Arc mRNA distribution in DG

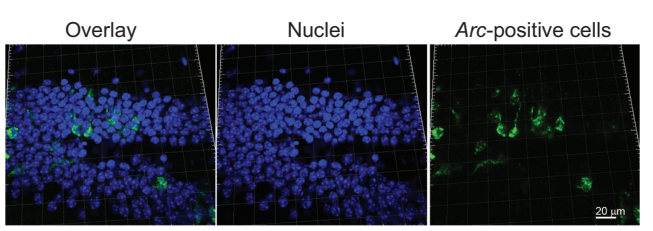

B Reconstruction

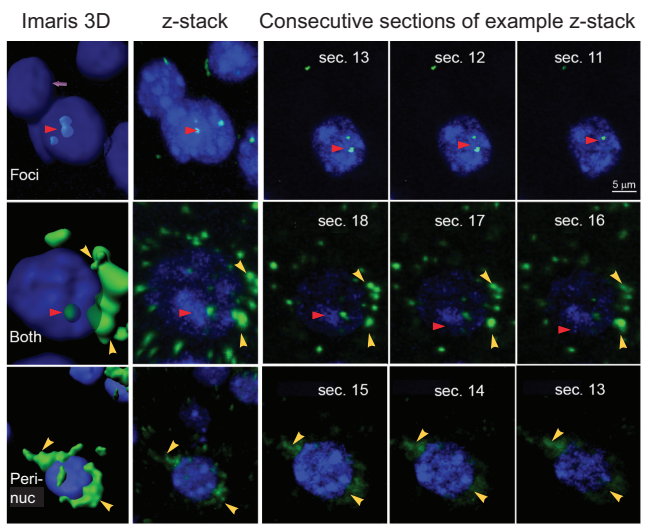

Figure 2. Neuron classification based on cellular compartmental expression of $\operatorname{Arc}$. (A) The typical Arc mRNA distribution in the dentate gyrus (DG) at $30 \mathrm{~min}$ after $5 \mathrm{~min}$ sound stimulation, providing a positive control brain area for confocal microscopy. Blue represents DAPI staining of nuclei: green represents $\operatorname{Arc} / \operatorname{Arg} 3.1$ mRNA expression. (B) (Left column) 3D surface reconstructions by IMARIS. Blue represents DAPI staining of nuclei; green represents $\operatorname{Arc} / \operatorname{Arg} 3.1$ mRNA expression. Arc-positive neurons were classified as "Foci-only" (red arrow) if they contained intranuclear fluorescent surfaces only (top); "Perinuc-only" if they contained perinuclear fluorescent surfaces (yellow arrow) only (bottom); and "Both" if they had intranuclear and perinuclear surfaces overlapping DAPI surface (middle). (Middle column) Confocal $z$-stack (63x magnification) of the corresponding tissue section $(20 \mu \mathrm{m} ; \sim 30-34$ optical sections) subjected to FISH. (Right three columns) Sequential sections of the $z$-stack showing "Foci-only" (top, sections 13-12-11), "Perinuc-only" (bottom, sections 18-17-16), and "Both" (middle, sections 15-14-13).

surfaces were not generated, and instead we manually counted DAPI nuclei and looked for areas of Arc fluorescence on consecutive planes of each $z$-stack (13 z-stacks; 777 cells; 8 animals). These manual counts (M) of Arc-positive (Foci-only, Perinuc-only, and Both) cells were compared with those determined by our 3D-assisted protocol (see Materials and Methods, Fig. 3A). For a nucleus to be designated as having Arc foci by the manual counting method, it had to exhibit Arc fluorescence in at least three consecutive planes, while cells with Arc signal spread around the nucleus were designated as having perinuclear cytoplasmic expression. Additionally, we compared our 3D-calculations for the same $z$-stacks using two different thresholds, with Ims2's threshold for Arc signal fluorescence increased by $10 \%$.

Even though there were not large mean differences in the percentages of cells counted by the two different methods (Fig. 3A), there were subtle but systematic differences apparent from paired comparisons. In particular, relative to the manual counts, small points of $A r c$ fluorescence within nuclei tended to be slightly overcounted as foci of $A r c$ by the 3D reconstruction ( 1\%, Fig. 3B, Foci-only, Ims1-M). This could be explained by the intensity threshold used for generating the 3D surfaces, since an increase of $10 \%$ in the threshold decreased the foci-only counts and eliminated the discrepancy between the 3D versus manual counting methods (Fig. 3B, Foci-only, Ims2-M). However, changing this threshold undercounted (albeit not significantly) neurons with
Both foci and perinuclear cytoplasmic expression (Fig. 3B, Both, Ims2-Ims1, and Ims2-M), resulting in an overall significant undercount for Arc-positive neurons (Fig. 3B, Arc-positive, Ims2-Ims1, and Ims2-M). No effect on Perinuc-only counts was found for such a change in threshold, consistent with those neurons' expression primarily being determined by large regions of Arc fluorescence. These results imply that there are tradeoffs in choosing thresholds for what is considered nonbackground expression. Importantly, the use of the computer-generated 3D surfaces makes these tradeoffs objective and explicit rather than subjective, and should enhance reproducibility of Arc counting. In any case, for all analyses presented here, cell count percentages based on 3D surfaces are compared across groups treated equally, minimizing the effect that any systemic bias would have in altering our conclusions.

Moving beyond methodological validation, we turned next to measuring expression differences dependent on familiarity with the acoustic features of pup USVs. A key acoustic feature characterizing the pup USV category is call frequency (Liu et al. 2003), a parameter that maternal mice can use as a basis for their categorical perception of USVs (Ehret and Haack 1982). We therefore started by asking whether playback of pure tones at $65 \mathrm{kHz}$ (Fig. 1C, left), the approximate frequency most commonly found in natural CBA/CaJ mouse pup calls (Liu et al. 2003), would induce Arc expression differently in core auditory cortical neurons of Dams versus Vrgs. The stimulus consisted of randomly generated sequence of $65 \mathrm{kHz}$ pure tone-pip bouts, with temporal properties (pip duration, intercall interval, interbout interval, and number of calls in a bout) spanning their ranges found in natural USV bouts (Fig. 1B, center). For both animal groups (Dam and Vrg), this particular synthetic tonal stimulus had never been encountered previously, though for the Dam, the tone frequency's closeness to natural USVs could affect its perception as pup-like (Ehret \& Haack 1982). After animals were given a 4-h period of silence in an anechoic chamber (Fig. 1B), they were either held in silence for an additional $30 \mathrm{~min}$ (Silence group), or were played a 5-min, $65 \mathrm{kHz}$ bout of tone stimuli before being sacrificed immediately ( $0 \mathrm{~min})$, or after a delay in silence (30 min).

Silence itself was associated with $\sim 16 \%$ Arc-positive expression (Foci-only + Both + Perinuc-only) in auditory cortex in both Dams and Vrgs, indicating a relatively high basal level of Arc expression in cortical layers III-VI (Fig. 4A, left bars). Tonal $65 \mathrm{kHz}$ stimulation led to a main effect of time in increasing the percentage of total Arc-positive neurons (time: $F_{(2,113)}=69.53, P=2.1 \times$ $10^{-20}, 2 \times 3$ ANOVA). However, there was no main effect of animal group and no interaction between time and animal group (animal: $F_{(1,113)}=0.07, P=0.80$; time $\times$ animal: $F_{(2,113)}=0.31, P=0.73,2 \times 3$ ANOVA). By the 30-min post-stimulus time point, Arc mRNA was expressed in nearly $60 \%$ of core auditory cortical neurons irrespective of animal group (Fig. 4A, right bars), affirming sound-induced expression of Arc. Significant post hoc comparisons are shown in Figure 4A.

For cells expressing Arc only as Foci in the nucleus (Fig. 4B), 65 $\mathrm{kHz}$ tone stimulation led to a main effect of time but not animal group, and only a trending interaction (time: $F_{(2,113)}=4.85, P=$ 0.0096; animal: $F_{(1,113)}=2.19, P=0.14$; time $\times$ animal: $F_{(2,113)}=$ $2.86, P=0.06,2 \times 3$ ANOVA). At the 0 -min time point (Fig. $4 \mathrm{~B}$, middle bars), the sound had elevated Foci-only expression significantly in Vrgs $(P=0.0138$, Tukey) and was trending in Dams $(P=0.071$, Tukey). Intriguingly, the Foci-only expression in Vrgs increased by 30-min time point compared with silence level $(P=0.0185$, Tukey), but no such increase was observed in Dams $(P=1.0$, Tukey).

Next, the percentage of neurons with Arc expressed only in the perinuclear cytoplasm following $65 \mathrm{kHz}$ stimulation showed a significant main effect of time, animal group, and interaction 


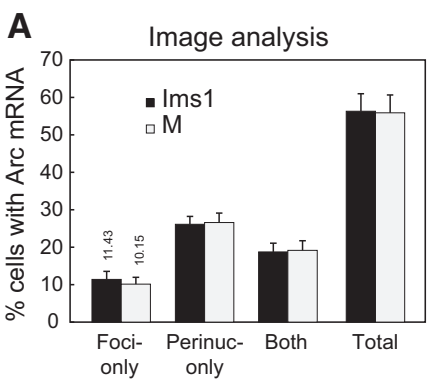

B

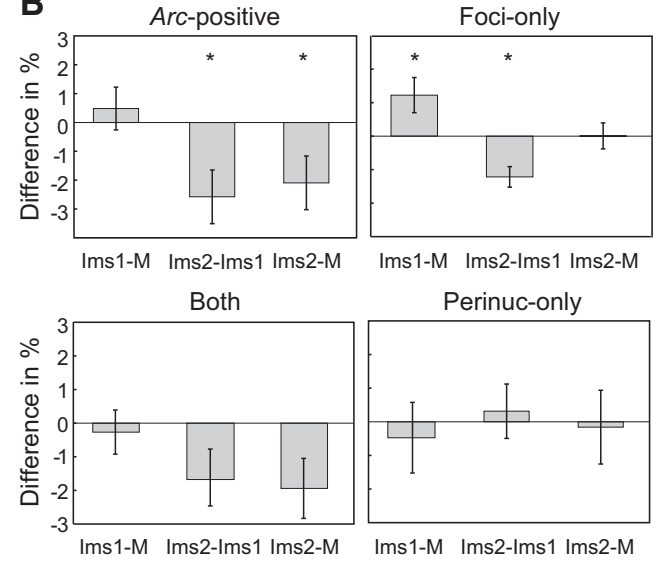

Figure 3. Comparative analysis of 3D-IMARIS and manual count of Arc expression in auditory cortex. (A) Comparison of 3D surface-assisted cell compartmental counts (Ims1, black bar) to manual counts (M, white bar). Percentage reflects the fraction of cells in a $z$-stack classified as having Arc mRNA in the specified compartment(s) (13 Z-stacks, 777 cells). No significant differences, except marginally for Foci-only cells (no multiple comparison correction). Ims1 versus $M$ paired $t$-test: Foci-only, $P=$ 0.045; Perinuc-only, $P=0.659$; Both, $P=0.54$; Total, $P=0.59$. (B) Paired comparisons of compartmental cell counts. Comparisons of Ims1, M, and Ims2 (10\% higher fluorescence threshold for 3D Imaris counts) suggest that increasing the fluorescence threshold for 3D surfaces reduces the number of Foci-only neurons closer to manual counts, but reduces the overall number of Arc-positive neurons because fewer neurons are classified as having Both Foci and Perinuc expression. Ims2 vs Ims1: Foci-only, $P=0.003$; Perinuc-only, 0.76 ; Both, $P=0.42$; Total, $P=0.023$. Ims2 versus $\mathrm{M}$ : Foci-only, $P=$ 0.95 ; Perinuc-only, 0.56; Both, $P=0.27$; Total, $P=0.05$. Asterisks $\left(^{*}\right)$ indicate significant paired $t$-tests $(P<0.05)$. Error bars represent standard error.

between time and animal group (Fig. $4 \mathrm{C}$; time: $F_{(2,113)}=67.64, P=$ $2.1 \times 10^{-20}$; animal: $F_{(1,113)}=5.61, P=0.0195$; time $\times$ animal: $F_{(2,113)}=7.69, P=0.0007,2 \times 3$ ANOVA). At 0 min immediately after the stimulation, Perinuc-only expression was low, and did not significantly differ between the groups ( $\operatorname{Vrg} 0 \mathrm{~min}: 3.9 \pm 2.2 \%$, Dam 0 $\min : 3.3 \pm 2.5 \%, P=1.0$, Tukey). By 30 -min though, the percentage of Perinuc-only neurons rose in both animal groups, but Dams had a higher percentage compared with Vrgs (Dam: $30.9 \pm 2.5 \%$, Vrg: $17.6 \pm 2.2 \%, P=2.7 \times 10^{-4}$, Tukey). Hence, even though total Arc-positive expression did not differ between animal groups at any time point (Fig. 4A), the cell compartmental composition of this total expression did (Fig. 4B, C). Under the assumption that $65 \mathrm{kHz}$ tones sound sufficiently like pup USVs to mothers (Ehret and Haack 1982), this difference in expression is consistent with the interpretation that a larger population of neurons with Perinuc-only Arc expression provides a molecular trace of whether new exemplars sound more familiar (Ivanova et al. 2011).

However, the fact that the expression difference was observed at the 30-min but not at 0-min time point raises some questions about the above interpretation, since we previously observed an increase in Perinuc-only expression 0-min after rehearing a (slightly longer) 10-min familiar stimulus (Ivanova et al. 2011). Therefore, as an independent test, our next experiment exposed animals to $5 \mathrm{~min}$ of either prerecorded pup USVs (Fig. 1B, center), or to the control, microphone noise (Nse) from the record-
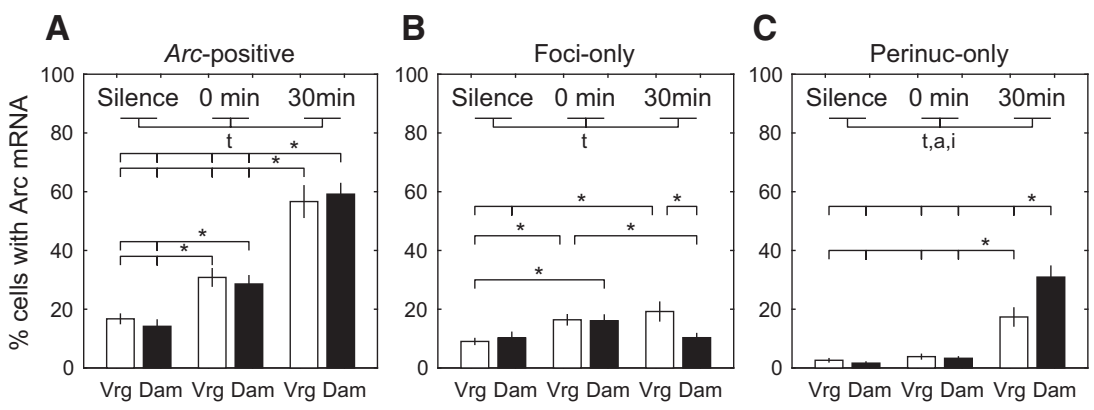

Figure 4. $\operatorname{Arc~mRNA}$ induction in core auditory cortex $0 \mathrm{~min}$ or $30 \mathrm{~min}$ after playback of 5 min of 65 $\mathrm{kHz}$ pure tone sequences. Neurons in layers III-VI of core auditory cortex were classified as Arc-positive (A), Foci-only (B), and Perinuc-only (C) for Vrgs (white bar, $23 z$-stack images) and Dams (black bar, 22 $z$-stacks) at 0 -min after stimulation, and for Vrgs (18 $z$-stacks) and Dams (18 $z$-stacks) at 30-min after stimulation. First two left bars in each panel shows Arc-labeled cells after $4.5 \mathrm{~h}$ silence. Of note, 65 $\mathrm{kHz}$ pure tone induces greater Foci-only expression in Dams and Vrgs immediately after playback tone, without any differences between groups. At $30 \mathrm{~min}$, the percentage of Foci neurons in Dams $(10.3 \pm 1.6 \%)$ becomes comparable to the silence level $(9.56 \pm 0.8 \%)$, but the percentage of Foci in Vrgs remains larger than in Dams ( $\operatorname{Vrg} 19.2 \pm 3.4 \%, P=0.026$ ). Tone models of pup USVs induce greater Perinuc-only expression in Dams versus Vrgs, but only at $30 \mathrm{~min}$, without any differences in overall Arc-positive expression. Significance in the two-way ANOVA is indicated for main effects of time $(t)$, animal group (a), or interactions between the two (i). Asterisks $\left(^{*}\right)$ indicate significant multiple comparison-corrected post hoc tests $(P<0.05)$. Error bars represent standard error. 

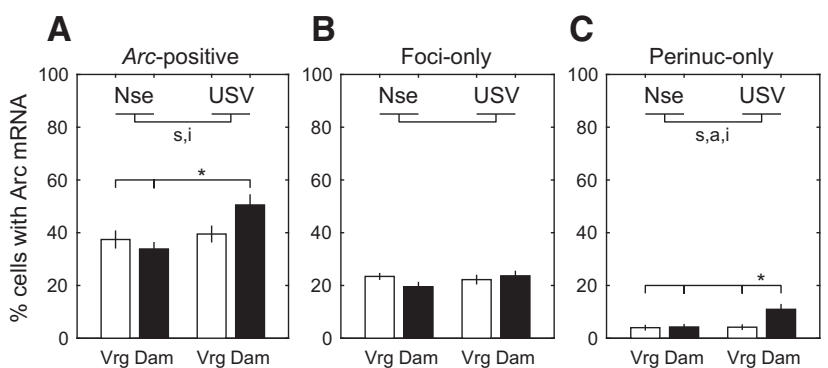

Figure 5. Arc mRNA expression in core auditory cortex 0 min after playback of 5 min of Nse or USV. Neurons in layers III-VI of core auditory cortex were classified as $\operatorname{Arc-positive~}(A)$, Foci-only $(B)$, and Perinuc-only (C) for Vrgs (20 z-stack images) and Dams (20 Z-stacks) exposed to Nse, and for Vrgs (19 z-stacks) and Dams (22 z-stacks) listening to USV. Natural pup USV induces greater Perinuc-only expression in Dams versus Vrgs, which cannot be explained by a response to microphone noise in the recording. Also after USV stimulation Dams had significantly higher level of Arc-positive cells versus Nse, and versus Vrgs after Nse. Significance in two-way ANOVA is indicated for main effects of stimulus (s), animal group (a), or interactions between the two (i). Asterisks $\left(^{*}\right)$ indicate significant multiple comparison-corrected post hoc tests $(P<0.05)$. Error bars represent standard error.

the animal differences observed for Perinuc-only expression elicited by the USV stimulus must be due to the presence of natural USVs in the recording. Since this was found as quickly as $5 \mathrm{~min}$ after the onset of sound stimulation, our data are consistent with the possibility that the time course of Arc mRNA expression and/or detectability in core auditory cortex is accelerated when acoustically realistic, familiar stimuli are heard.

Alternatively, one might argue that the differences observed between Dams and Vrgs might simply be due to dissimilarities in their history of reproductive hormones, even though at the postweaning time point of sound playback here, both Dams and Vrgs would be cycling normally. It may also be that pup USVs are far more salient to Dams than to Vrgs, irrespective to whether they are familiar or not. To address these possibilities within our natural paradigm, we next investigated a third animal group of Cocs, that is, virgin cocarers. These females gain the same duration of pup care and USV exposure as Dams, but do not experience the hormones of pregnancy, parturition, and lactation to the same degree (Fig. 1A). Furthermore, pup USVs apparently have a diminished degree of behavioral salience for Cocs compared with Dams after pups are weaned (Ehret and Koch 1989; Lin et al. 2013). Hence, Cocs and Dams should be similar in terms of their level of acoustic familiarity with pup USVs through their common pup care experience, but differ in their hormonal trajectories and degree of pup USV salience post-weaning, which for post-weaning Cocs are more similar to Vrgs.

To determine whether Cocs are more similar to Dams or Vrgs in their pattern of Arc expression after hearing pup USVs, we played the natural calls to animals from all three groups and sacrificed them after a 30-min delay (Guzowski et al. 2001; Khodadad et al. 2015). As with the $65 \mathrm{kHz}$ tones, Arc mRNA was expressed in nearly $60 \%$ of core auditory cortical neurons irrespective of an animal group, and there were no significant differences (Fig. 6A; $F_{(2,75)}=$ $0.99, P=0.38,1 \times 3$ ANOVA). However, at the compartmental level, animal group significantly modulated both the percentage of Foci-only (Fig. 6B; $F_{(2,75)}=4.48, \quad P=0.01,1 \times 3$ ANOVA) and Perinuc-only (Fig. 6C; $F_{(2,75)}=5.89, P=0.0042,1 \times 3$ ANOVA) expression. The percentage of Foci-only cells in Dams $(10.5 \pm 1.4 \%)$ was similar with their level for silence, and was not different from that seen $30 \mathrm{~min}$ after $65 \mathrm{kHz}$ playback (Fig. 4B, 10.3 \pm $1.6 \%)$. In contrast, Vrgs $(16.1 \pm 1.3 \%)$ had the highest Foci-only percentage of all animal groups, which was significantly larger than for Dams $(P=0.005$ Fisher's), but not than Coc $(13.8 \pm$ $1.3 \%, P=0.26$, Fisher's).

Furthermore, after a 30-min delay Vrgs $(21.9 \pm 1.8 \%)$ had a significantly lower percentage of Perinuc-only expressing neurons compared with Dams $(30.1 \pm 1.6 \% ; P=0.0011$, Fisher's) and Cocs $(26.9 \pm 1.7 \%, P=0.046$, Fisher's). Hence the pattern of compartmental Arc expression in Cocs appeared to more closely align with that of Dams, suggesting that neither a maternal hormonal trajectory nor sustained salience of pup USVs are needed to be able to see a bias toward greater Perinuc-only Arc expression (summarized in Fig. 6, bottom). This result therefore supports the hypothesis that compartmental Arc expression in core auditory cortical neurons can provide a molecular trace of the experiencedependent familiarity of a sound category, even when the exact exemplars heard are novel.

Finally, pooling sound-stimulated data across animal groups, we found that a sound as short as 5 min induces changes in the transcription of Arc mRNA in the auditory cortex, as shown by averaging compartmental expression across all stimuli (Fig. 7A; time: $F_{(2,314)}=114.46, P=3.3 \times 10^{-45}, 1 \times 3$ ANOVA). These changes are in fact observed for each of the stimuli (Fig 7B): Tone (time: $F_{(2,131)}=$ 84.17, $P=3.1 \times 10^{-24}, 1 \times 3$ ANOVA), Noise (time: $F_{(2,112)}=122.61$, $P=6.2 \times 10^{-29}, 1 \times 3$ ANOVA), and USVs (time: $F_{(2,167)}=123.14, P=$ $1.4 \times 10^{-34}, 1 \times 3$ ANOVA). These results suggest that brief sound exposures in general recruit a continuing cascade of new Arc mRNA (reflected in the combined Both + Foci-only category) in auditory cortex extending beyond the typical $\sim 10$ min window after experience to which hippocampal CA1 Arc expression is usually confined. Apparently, the time course of Arc expression in this
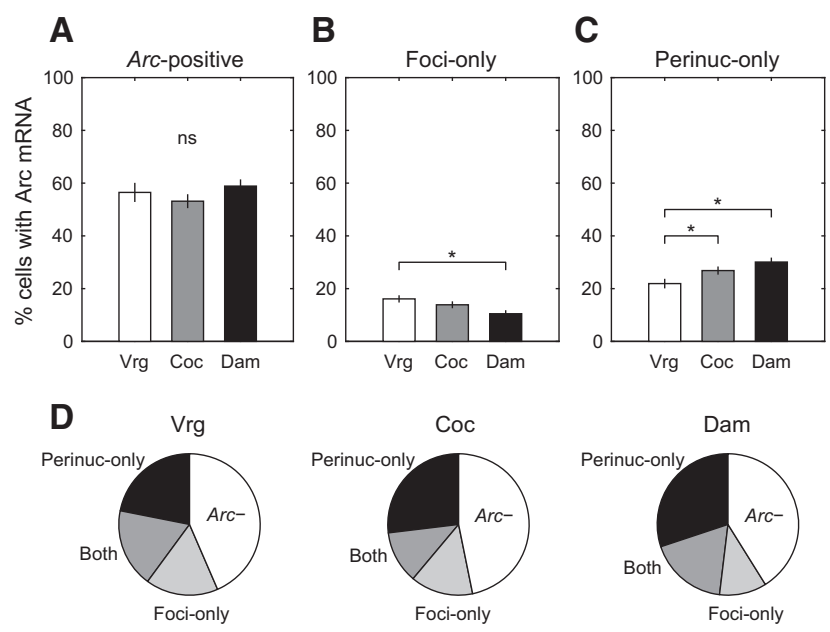

Figure 6. Pup care experience and not simply maternal hormonal history and call salience biases Perinuc-only expression of Arc mRNA in core auditory cortex 30 min after playback of 5 min of USV. Neurons in layers III-VI of core auditory cortex were classified as $\operatorname{Arc-positive~}(A)$, Foci-only (B), and Perinuc-only (C) for Vrgs (25 z-stack images), Cocs (26 z-stacks), and Dams (28 z-stacks). Coc mice exhibit a pattern of compartmental Arc expression that is not significantly different from Dams, but is significantly different from Vrgs. (D) Recruitment of Arc-transcribing neurons is accelerated by hearing exemplars of a familiar stimulus category. Sacrificing Vrgs 30-min after playback of USV leads to a steady rate of recruitment of new neurons transcribing $A r c$, as seen in the roughly equal proportion of neurons classified as Perinuc-only (earliest response, black), Both (intermediate response, dark gray) and Foci-only (most recent response, light gray). Prior experience with vocalizing pups biases the recruitment toward earlier transcription, so that a larger proportion of neurons are classified as Perinuc-only (black) in Cocs and Dams. Asterisks $\left({ }^{*}\right)$ indicate significant multiple comparison-corrected post hoc tests $(P<0.05)$. Error bars represent standard error. 
A

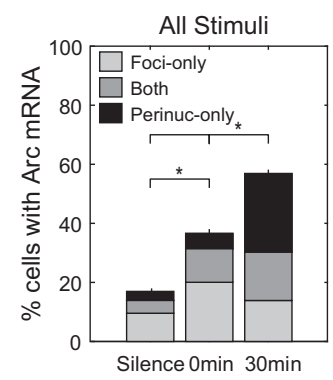

B

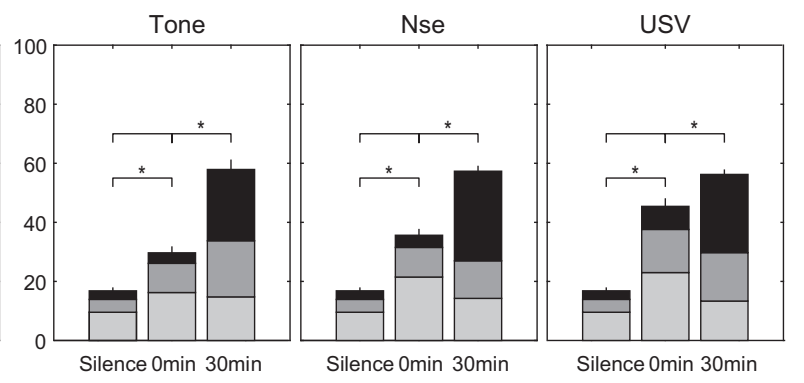

Figure 7. (A) The overall effect of 5-min sound playback on the Arc mRNA expression. (A) Sound playback, regardless of the specific sound, or one's familiarity with it (i.e., pooling Dam, $\mathrm{Vrg}, \mathrm{Coc}$ ) leads to the recruitment of new neurons transcribing Arc versus silence (51 Z-stack images) immediately after playback (0-min, 128 Z-stack images). Up to $\sim 40 \%$ of all neurons become Arc-positive, most of them classified as Foci-only (new Arc transcription, light gray). Thirty minutes after playback up to $\sim 60 \%$ of all neurons are Arc-positive (138 Z-stack images), most of them are classified as Perinuc-only (black), and Both (dark gray); the proportion of cells with only new Arc transcription (Foci-only) returned to silence level. (B) Time-dependent expression for each specific stimulus, regardless of familiarity. Asterisks $\left(^{*}\right)$ indicate significant multiple comparison-corrected post hoc tests in the total Arc-positive expression $(P<0.05)$. Error bars represent standard error.

sensory cortical network can be different from that seen in the hippocampus.

\section{Discussion}

Neural plasticity to encode behaviorally relevant stimuli is a wellestablished mechanism for sensory learning and memory, yet studies of this have generally overlooked the question of how variable stimuli within a learned category engage plasticity mechanisms. A balance has to be found between plasticity to incorporate stimulus features of new exemplars, and stability to maintain previously learned representations. This could be achieved if plasticity mechanisms themselves were sensitive to the history of prior experience with similar stimuli, even when those stimuli are not identical to those experienced earlier. Here we have shown that the intracellular compartmentalization of a key synaptic plasticity effector immediate early gene, $\mathrm{Arc} / \mathrm{Arg} 3.1$, in core auditory cortex exhibits such flexibility even if animals hear new examples of familiar natural vocal category.

An important methodological point is that our compartmental analysis utilized manually supervised, computer-generated 3D surfaces (Fogarty et al. 2013; Lee et al. 2015) to classify cells based on the location where Arc mRNA is expressed, which differs from prior approaches (Chawla et al. 2004; Ramirez-Amaya et al. 2005). Our analysis is based on $z$-stack confocal imaging of DAPI nuclear and Arc fluorescence staining, as with all compartmental analysis methods. However, our image analysis software then allows the generation (guided by manual verification) of artificial $3 \mathrm{D}$ surfaces for both, using parameters tailored to approximate the real fluorescence seen in the original z-stacks. These artificial surfaces greatly simplify a less biased definition of the nuclear edge, especially in cases where this edge is not well delineated in all layers of the original $z$-stack. The ability to rotate each nuclear surface to verify the relative location of its Arc fluorescence also enhances confidence in the compartmental classification. Moreover, even though we, like others (Chawla et al. 2004; Ramirez-Amaya et al. 2005), cannot assign Arc fluorescence far from the nucleus as belonging to a specific neuron without the use of a marker for cell membranes, the use of 3D surfaces at least provides a systematic way to capture perinuclear Arc expression from the overlap of $A r c$ and nuclear surfaces. The importance of such an objective method is apparent from the fact that increasing/decreasing the quantitative fluorescence threshold for $3 \mathrm{D}$ surface generation can bias ( $\sim 1 \%$ discrepancy) the compartmental percentages toward equivalent/higher Foci-only and simultaneously lower/equivalent Both expression relative to manual counts (Fig. 3B). While every method has its advantages and disadvantages, the key point here is that our comparisons across animal groups were analyzed by the same methods in a blind fashion.

In our study, we used the ultrasonic communication system between mouse pups and adult female mice to test whether prior experience with vocalizing pups alters the pattern of Arc expression elicited by both synthetic and natural exemplars of the pup USV category. In none of our experiments was the "total" percentage of Arc-positive neurons in core auditory cortex sensitive to prior experience hearing and/or interacting with mouse pups. Critically though, the compartmental expression of Arc was. When listening to novel exemplars of natural calls, mothers show a more rapid and sustained increase in the population of neurons with Perinuc-only expression compared with pup-naïve virgins (Figs 5C, 6C), which could arise from $A r c$ mRNA being generated either earlier or with much greater magnitude. When listening to pure tone models of pup USVs, which are still salient to mothers (Ehret and Haack 1982) though acoustically less similar to the natural pup USVs they heard during pup rearing, a higher Perinuc-only percentage was again observed, albeit after a longer delay (Fig. 4C). Hence, compartmental Arc expression is sensitive to prior experience with the "category" of pup USVs, extending our previous result based on replaying "identical" familiar stimuli (Ivanova et al. 2011).

As a further test of whether this category familiarity, and not just a mother's hormonal history and/or the immediate salience of the USVs, biases the Perinuc-only expression, we also played back natural USVs to cocaring females after pups had been weaned. Cocarers also showed a higher Perinuc-only population than naïve virgins (Fig. 5C). Altogether, our results are consistent with the interpretation that prior experience hearing exemplars of a sound category helps a larger population of core auditory cortical neurons to accumulate Arc mRNA in just the perinuclear cytoplasmic compartment once new category exemplars are heard. We speculate that the engagement of this molecular mechanism for plasticity is therefore itself plastic and sensitive to experience.

Our results fit within an emerging conceptual framework for understanding Arc's function in modifying synaptic connectivity after experience, and extend this framework by considering new sensory experiences that are congruent with but not identical to previous experiences. Completely novel stimuli can trigger a temporally extended program of Arc transcription within the nuclei of sensory cortical and hippocampal neurons, typically peaking 1530 min after a brief experience, depending on the type of sensory experience and brain area (Guzowski et al. 2001; Kelly and Deadwyler 2002, 2003; Ramirez-Amaya et al. 2013; Khodadad et al. 2015; Nakayama et al. 2015). In core auditory cortex, this is seen within 5 min of the onset of an unfamiliar stimulus category through the $\sim 30 \%$ of $A r c$-positive neurons in Vrgs listening to pure tones (Fig. 4A, middle white bar), Vrgs and Dams listening to noise (Fig. 5A, left white and black bars, respectively), or Vrgs listening to pup USVs (Fig. 5A, right white bar). According to the current understanding of Arc kinetics (Vazdarjanova et al. 2002), initially transcribed $A r c$ mRNA is fully transported from the nucleus into 
the perinuclear cytoplasm by $\sim 30$ min later, and is being shuttled into the dendrites or degraded (Das et al. 2003). Since cells with Arc mRNA only in the dendrites or disconnected from the nuclear membrane were not counted by our methods, an initial $27 \%$ (35\%) Foci-positive (Foci-only + Both) cell population in Vrgs seen for tone (pup USV) stimulation at 0 min became a population of only $17 \%$ (22\%) of Perinuc-only cells by $30 \mathrm{~min}$. This $\sim 37 \%$ reduction for both novel stimulus categories is consistent with decays that have previously been reported for this time point, suggesting that initial auditory cortical Arc expression in Vrgs for novel stimuli follows the well-characterized kinetics seen in hippocampus CA1 (Vazdarjanova et al. 2002).

Importantly though, in light of this self-consistent result for novel stimuli in Vrgs, the decays for natural pup and tonal model USVs in Dams, the experimental group with prior familiarity with pup USVs, are noticeably slower than in Vrgs. For $65-\mathrm{kHz}$ tone models (Fig. 4), Dams initially had 25\% Foci-positive (Foci-only + Both) cells at $0 \mathrm{~min}$, and a statistically indistinguishable $31 \%$ Perinuc-only cells by $30 \mathrm{~min}(P=0.23, t$-test). For pup USVs (Figs. 5C, 6C), an initial 39\% Foci-positive population at 0-min decayed to $30 \%$ at $30-\mathrm{min}$, a significant $23 \%$ decrease $(P<0.005, t$-test $)$. This smaller stimulus-dependent decay in mothers relative to virgins ( $23 \%$ versus $37 \%$ ) was a surprise-one that we did not initially hypothesize-but shows how Arc expression unfolds across the neural network based on prior experience (Abraham 2008). Hence, not only is the initial expression of Arc across the core auditory cortical population apparently faster, but it seems to decay more slowly when a sound category is somewhat familiar.

Furthermore, our data from the 30-min time point also show that sound-induced expression of Arc in naïve mice (Vrg and Coc) continues for longer than in Dams while listening to the tones (Fig. $4 \mathrm{~B}$, right bars) or USVs (Fig. 6B). Since silence itself produces no more than $10 \%$ Foci-only and $\sim 3 \%$ Perinuc-only neurons in auditory cortex (Fig. 7), with no difference between animal groups, comparable to our earlier study (Ivanova et al. 2011), we suggest that these neurons with newly transcribed Arc mRNA 30 min after sound presentation are likely responding (late) to the sound, and not simply exhibiting baseline transcription.

Why there may be a temporally extended window for recruiting more neurons to transcribe Arc is not entirely clear, but it may reflect neuronal diversity in different brain areas in the time constants for the molecular cascades that lead up to Arc transcription (Saha et al. 2011; Ramirez-Amaya et al. 2013). That diversity might, for example, arise from time constants that effectively vary with the strength of initial neuronal depolarization by stimuli, which presumably depends on how strongly a neuron is connected within the neural network ultimately driven by the stimulus (Sheng et al. 1990). Alternatively, permissive neuromodulatory inputs that are temporally diffuse ( $\mathrm{Gu} 2002)$ or delayed recurrent network activity (Yasuda et al. 2007) may gradually increase the population of neurons newly transcribing Arc. In this light, we recently found that muscimol injected into auditory cortex immediately after an auditory fear conditioning session impairs the consolidation of the acoustic cue, suggesting that nonstimulus locked auditory cortical neural firing after a sound experience is necessary for learning (Banerjee et al. 2017). Furthermore, the possibility for late transcription was also observed in the dentate gyrus (but not the CA1) of rats, where Arc transcription was sustained for hours following spatial exploration (Ramirez-Amaya et al. 2013). At the 30-min time point in core auditory cortex, the timeline of neuronal recruitment can be inferred from the compartmental expression (Fig. 2; Zelikowsky et al. 2014). The neurons expressing just "Perinuc-only" Arc must have been recruited earliest during the stimulation, with those showing "Both" nuclear and perinuclear cytoplasmic Arc and those showing "Foci-only" Arc mRNA being recruited progressively later, respectively. For a completely novel stimulus category, such as Vrgs listening to USVs, recruitment appears to be relatively steady over time based on the approximately equal proportions of the Arc-positive neural population in these different classes (Fig. 6, bottom, Vrg). Interestingly, recruitment appears capped at $\sim 60 \%$ of neurons in core auditory cortex, perhaps due to baseline, intrinsic connectivity, since Arc-positive expression appears generally limited to this irrespective of the stimulus (Fig. 4A, right bars; Fig. 6A).

The function of this Arc is presumed to help consolidate the auditory memory traces triggered by the sound so as to better reactivate relevant neurons when the same sound is encountered in the future. Through its role in weakening less active synapses (i.e., those that were not well-driven by the stimuli) in an activated neuron (Okuno et al. 2012), and consolidating memories associated with potentiated synapses (Guzowski et al. 2000; Messaoudi et al. 2007; Korb et al. 2013; Ramirez-Amaya et al. 2013), Arc is hypothesized to improve the signal to noise in the ability of stimulusdependent synaptic inputs to drive a neuron (Morin et al. 2015). At the same time, it may be paring away connectivity in neurons that are much less driven by the stimulus, consistent with a role for Arc in stimulus selectivity in the visual cortex (Wang et al. 2006).

These functions of Arc would ultimately alter how the population of neurons subsequently responds electrophysiologically to the presentation of the identical stimulus. Once such a "familiar" stimulus is encountered, many of the neurons that previously expressed Arc mRNA would now be better driven by the specific sound, and we speculate that there might be some nuclear history, perhaps in the form of epigenetic marks, that facilitate Arc transcription more rapidly. This could explain the early accumulation of Perinuc-only neurons in animals that heard sounds that were more familiar from prior experience (i.e., Dams and Cocs listening to USVs, Fig. 6, bottom, respectively). Alternatively or in addition, neurons from animals with prior experience might have more efficient processing or export of preexisting and/or newly induced $A r c$ mRNA (Johnson et al. 2009). Furthermore, the degree to which new exemplars of the same familiar category of sound engage an overlapping set of synapses that were previously potentiated by that sound category would determine how much of a familiarity effect would be observed in the Perinuc-only population. This could explain the late $30 \mathrm{~min}$, but not $0 \mathrm{~min}$, familiarity effect seen in Dams (compared with Vrgs) listening to the tonal models of pup USVs.

Our results support the view that the continual engagement of a plasticity gene such as Arc, even when a stimulus category is familiar, helps incorporate new information about a stimulus in the behavioral contexts it is encountered (Miyashita et al. 2009; Morin et al. 2015), much like the process of memory reconsolidation (Dudai 2012; Bozon et al. 2003). This could be an essential mechanism by which the cortex builds up a more robust representation of stimulus categories from different experiences with that category, allowing hippocampal mechanisms of pattern recognition to better recall those events, even when the stimuli are incomplete or degraded (Rolls 2016). Other paradigms have also found that familiar stimuli or environments still engage Arc expression, sometimes even more so than novel contexts (Miyashita et al. 2009; Ivanova et al. 2011; Morin et al. 2011). In our studies here, we presume that the Arc-positive expression in Dams listening to USVs was as high as in Vrgs because of the novel environment in which the sounds were played back (anechoic chamber, without actual pups). An interesting question to investigate in the future would be whether some repeated number of identical trials might lead to a saturation of what new information can be gained in an experience (habituation), leading eventually to a reduced percentage of neurons expressing Arc. This might perhaps explain why baseline home cage expression is generally low, and 
why a well-learned tone elicits less Arc expression (CarpenterHyland et al. 2010). In any case, irrespective of why the total number of neurons transcriptionally activated remains high in familiar conditions, our results provide new evidence at the population level that the expression of Arc mRNA is nevertheless altered between initial and later experiences. Therefore, this work underlines the possibility that Arc mRNA may play a role in the process of incorporating new stimulus information about already familiar sounds, and enabling the subtle refinement of existing sensorineural representations.

\section{Materials and Methods}

\section{Experimental design}

All procedures were approved by the Emory Institutional Animal Care and Use Committee. Experiments were performed on CBA/ CaJ female mice (15-16 wk old), which were housed at least two per cage in a colony under reversed 14-h light/10-h dark cycle. Animals had access to food and water ad libitum, with experiments conducted during their dark cycle.

Figure 1A illustrates our experimental timeline for each of three groups of adult female mice (3-4 animals per group). Primiparous Mothers (Dam), which were initially paired at the age of 9 wk with a male for mating and then separated into a new cage at least $10 \mathrm{~d}$ before pups were born, had at least $21 \mathrm{~d}$ of caring for their pups experience. Cocarers (Coc), which were added to a littermate Mother's home cage on the same day Mother was separated from the male, had the same full-term experience of caring for pups, but without physiological changes associated with pregnancy, parturition, and lactation. All Mothers and Cocarers were screened to check for successful retrieval of scattered, vocalizing pups in the home cage over a 10-min period on postnatal day P5 or P6. Pups were weaned when they reached P21, after which Mothers and Cocarers were separated into individual cages for 24 h. Naïve virgins (Vrg), which were adult female mice (agematched to Dam and $\mathrm{Coc}$ ) with neither mating experience nor physiological changes associated with pregnancy, parturition, and lactation, had no adult contact with pups. Virgin mice were group housed in the colony and placed into an individual home cage $24 \mathrm{~h}$ before experiments.

On a given experiment day, an individual mouse in its home cage was placed into a silent anechoic chamber $\left(44^{\prime \prime} \times 27^{\prime \prime} \times 24^{\prime \prime}\right.$, $\mathrm{W} \times \mathrm{D} \times \mathrm{H}$ inner dimensions, Acoustic Systems) for 4-h for habituation, followed by a 5-min test period of sound stimulation (Fig. 1B). Mice were decapitated immediately or with 30-min delay after sound stimulation, and brains were processed for in situ hybridization experiments targeting the immediate early gene Arc.

\section{Acoustic stimulation}

Sounds were generated by a TDT (Tucker Davis Technologies) RX6 digital signal processor at a sampling rate of 233 kilosamples/second, attenuated by a PA5 programmable attenuator (Tucker Davis Technologies), and played through an EMIT speaker (Infinity, EMIT). Acoustic stimuli included 5-min bouts of ultrasonic pure tones, prerecorded ultrasonic pup vocalizations (USV) and broadband intrinsic microphone recording noise (Nse). Pure tones (Fig. 1B, left) consisted of dynamic bouts of a random number of $65 \mathrm{kHz}$ tone pips (mean of 4, range 2-6), with random durations $(60 \pm 24 \mathrm{msec}$, mean \pm standard deviation) and inter-tone intervals (206 $\pm 49 \mathrm{msec}$ ) within a bout, and random interbout intervals $(755 \pm 146 \mathrm{msec}$ between the end of one bout and the start of the next). This provided a tonal model of mouse pup calls with a frequency matching with the most commonly found in these USVs (Liu et al. 2003). Natural pup USVs (Fig. 1B, center) were previously recorded from P6-7 pups (Liu et al. 2003) using a 1/4" calibrated microphone (Bruel and Kjaer). The 5-min recording was selected so that the mean frequency of detected pup calls in the sound file was close to $65 \mathrm{kHz}$. The recording was high-pass filtered above $25 \mathrm{kHz}$. And third (Fig. 1B, right), 5-min of microphone thermal prerecorded noise (wide-bandwidth, arising from the intrinsic noise floor of the microphone) was used as a control sound for the USV recording. Pup calls were removed from the recording, and those segments were replaced with random quiet background noise segments from the remaining recording. The resulting sound file was high-pass filtered above $25 \mathrm{kHz}$ and scaled by the same factor as the original USV to match its background noise.

\section{Tissue processing and fluorescent in situ hybridization (FISH)}

Immediately after decapitation, brains were rapidly removed, covered with OCT media (VWR International), and frozen promptly in liquid nitrogen. Frozen brains were stored at $-80^{\circ} \mathrm{C}$ prior to cryosectioning. Serial $20 \mu \mathrm{m}$ coronal sections cut by cryostat (Leica) were captured on Super-frost slides (Fisher Scientific), and stored at $-80^{\circ} \mathrm{C}$ until fluorescent in situ hybridization (FISH). FISH was performed on frozen slide-mounted brain sections following protocols previously described in detail elsewhere (Guzowski et al. 1999; Muddashetty et al. 2007; Ivanova et al. 2011). Briefly, Digoxigenin-labeled Arc antisense and sense riboprobes (NCBI accession number NM_018790.2, nucleotides 273-1369) were prepared using a commercial kit (Roche Molecular Biochemicals). After riboprobe hybridization $\left(16 \mathrm{~h}, 56^{\circ} \mathrm{C}\right)$, slides were treated with RNaseA $(10 \mathrm{mg} / \mathrm{mL})$ and $3 \%$ hydrogen peroxide, and followed by incubation in block buffer containing anti-digoxigenin-POD, Fab fragments (Roche Diadnostics). Arc probes were detected with TSA-Direct Cyanine-3 fluorescence amplification kit (TSA Amp Kit, PerkinElmer). Nuclei were counterstained with 4',6-diamidino-2-phenylindole (DAPI, Electron Microscopy Sciences).

\section{Confocal microscopy and cell counting}

Image acquisition was performed using a Zeiss LSM 510 (Carl Zeiss Jena $\mathrm{GmbH}$ ) or in the Olympus FV1000 (FluoViewFV1000 Spectral Confocal Laser Scanning Microscope, Olympus Corporation of the Americas) confocal microscope. Coronal sections were matched to a standard mouse atlas (Paxinos and Franklin 2001) to identify the anatomically labeled primary auditory cortex (Au1); sections fell between -2.30 and $-2.80 \mathrm{~mm}$ relative to Bregma. The regions of auditory cortex and cortical layers in the coronal slices were identified based on nuclear DAPI staining with a $10 \times$ objective (Anderson et al. 2009). Then, using a $63 \times$ oil objective, confocal $z$-stacks composed of $0.5 \mu \mathrm{m}$ thick optical sections were collected through the regions of interest. All images were saved in a 12 bit TIFF format at $512 \times 512$ or $1024 \times 1024$ pixels. The dentate gyrus was used as a positive control area for confocal microscopy, in which a small number of Arc mRNA strongly labeled cells can always be detected. By $30 \mathrm{~min}$, Arc mRNA was located predominantly within the perinuclear cytoplasm, as expected based on prior literature from the hippocampus (Guzowski et al. 1999; Vazdarjanova et al. 2002; Vazdarjanova and Guzowski 2004). The Figure 2A shows a typical Arc mRNA distribution in DG at 30 min delay after 5 min sound stimulation.

Our analysis focused on cortical layers corresponding to the thalamorecipient layers III-IV and infragranular layers V-VI in auditory cortex (Cruikshank et al. 2002; Winer et al. 2005). For each layer (layer III-IV and V-VI), usually two nonoverlapping z-stacks at a given cortical depth were imaged per slide. A confocal $z$-stack consisted of $\sim 30-34$ optical sections, spaced $0.5 \mu \mathrm{m}$ apart. Typically 12-24 z-stacks were collected per experimental group. Arc mRNA fluorescence was analyzed in images of sections incubated with sense or anti-sense riboprobes. Sense-riboprobe images, which usually showed very weak intensity, were used to confirm the specificity of the probe to bind Arc mRNA. A 3D image volume was reconstructed from sequential $z$-sections and analyses were performed with Imaris software (Bitplane Scientific Software), which generated artificial surfaces around both DAPI-stained nuclei and fluorescently labeled Arc mRNA that allowed for a less biased estimate of the physical extent of these signals. Artificial surfaces were created by thresholding absolute intensity and smoothing, and were visually compared with the original $z$-stack. The software's nucleus diameter setting was initially determined by using the line tool to measure the diameter of a random subset 
of DAPI-nuclei found in the confocal stack, and using the interactive DAPI-channel software histogram to select a threshold to include as many nuclei as possible while excluding background. The segmentation of nuclear objects was performed by "Seed" algorithm, with seed point diameter set to this minimum diameter (typically $5 \mu \mathrm{m}$ ), and the result was manually verified by visually comparing against the original $z$-stack. In addition, we manually split large clusters of merged surfaces that actually represent closely spaced distinct nuclei. Nuclei that were cut off at the edges of the $z$-stack were removed, and only whole nuclei were analyzed. We also manually removed presumed glial cells, which had much smaller nuclei, stained strongly with DAPI, and did not express Arc (Chawla et al. 2004). The average neuron-like 3D-DAPI surfaces in each image analyzed was $59.7 \pm 1.67$ (mean \pm standard error), and at $20.9 \pm 0.8$ images per animal group, we had on average $1248.9 \pm 68.5$ nuclei per animal group. All cells were classified as positive or negative for $A r c$-signal by making nuclei translucent (the transparency of a DAPI-channel is altered by adjusting the opacity to $56 \%$ ), and rotating the object $360^{\circ}$ horizontally and $180^{\circ}$ vertically to change the viewing angle.

For Arc mRNA analysis, the green channel (Alexa 488) was selected as the source, and artificial surfaces were created by thresholding absolute intensity. In order for a cell to be classified as Arc-positive, we required that an Arc-3D surface be in contact with its DAPI-surface. Neurons distributed across the mouse auditory cortex showed a characteristic intranuclear signal, which consisted of one or two discrete foci of intense fluorescence. Cells that contained two clear intranuclear Arc surfaces, which had to have a round, smooth shape, and size of at least $\sim 2.5 \mu \mathrm{m}$ diameter, were counted and classified as "Foci." However if only one Arc-3D surface was detected, but had a smooth shape and size of around $\sim 4.0-4.5 \mu \mathrm{m}$, we compared the 3D surface to the original $z$-stack to validate such an image before including that nucleus into our Arc-positive counts. In addition to cells with intranuclear foci, we observed cells with two other distinct staining profiles: cells with Arc mRNA perinuclear cytoplasmic staining only (Fig. 1A), and those with both intranuclear and perinuclear cytoplasmic staining. Cells that only exhibited diffuse, predominantly perinuclear (e.g., $\sim 70 \%$ of volume was outside the DAPI surface), Arc staining were classified as having Arc-perinuclear-cytoplasmic expression (Perinuc-only). Double-labeled cells that contained perinuclear and intranuclear staining of Arc were classified as "Both". Each of the three compartmental patterns were calculated as percentage of the overall DAPI-stained cells for each image. The sum of the percentages of cells expressing Arc mRNA (Foci-only, Both, Perinuc-only) totaled the percentage of $A r c$-positive neurons. Note that "Foci-only" and "Perinuc-only" should be distinguished from "Foci-positive" and "Perinuc-positive" cells, which were the sum of cells with "Foci-only" and "Perinuc-only," respectively, with "Both" expression. Data from layers III-IV and V-VI were combined, and averaged across the cortical layers III-VI for each group animals.

\section{Comparison of 3D surface-assisted quantification of Arc expression to manual counting}

To validate our 3D surface-assisted counting methods against existing manual counting methods, we separately counted cells in a subset of images using a reconstructed sequential confocal z-stack (Fig. 1B, top; Chawla et al. 2004). The manual image analysis was performed using the Slice View application in the IMARIS software. We used this application to view individual $z$-sections, and navigate to any position (XY, XZ, and $\mathrm{YZ}$ ) within each 3D stack. $Z$-stacks were then evaluated for Arc-positive (Foci-only, Both, or Perinuc-only) staining. The thresholds for foci size and intensity, and thresholds for perinuclear cytoplasmic distribution were set and equal to the threshold we used for 3D IMARIS. For a cell to be designated as having a "foci" of Arc expression in the nucleus, a minimum intensity threshold had to be present in consecutive planes; in most cases, this was at least three planes. For perinuclear labeling, cells with the Arc signal spread around the nucleus over at least three planes, were designated as having "perinuclear cytoplasmic" expression.

\section{Statistics}

Data were analyzed either by one-way or two-way ANOVA in MATLAB (Mathworks). In the case of two-way ANOVAs, post hoc tests were carried out by Tukey's honestly significant difference (HSD) to control for multiple comparisons. Differences were considered significant at $P<0.05$.

\section{Acknowledgments}

We thank Dr. Edgar Galido-Leon, Dr. Frank G. Lin, and Adam Willats for fruitful discussions. We thank the staff of Emory University Integrated Cellular Imaging Microscopy Core and especially Neil Anthony for his help in the analysis of 3D-images. We thank Xiaoyu Xu for help in the classification of Arc-positive cells. This work was supported in part by NIDCD R01DC008343 (R.C.L.), NIH training grant 5R90DA033462 (Y.J.K.) and the Emory University Integrated Cellular Imaging Microscopy Core of the Emory Neuroscience NINDS Core Facilities grant, P30NS055077.

\section{References}

Abraham WC. 2008. Metaplasticity: tuning synapses and networks for plasticity. Nat Rev Neurosci 9: 387-399.

Anderson LA, Christianson GB, Linden JF. 2009. Mouse auditory cortex differs from visual and somatosensory cortices in the laminar distribution of cytochrome oxidase and acetylcholinesterase. Brain Res 1252: $130-142$.

Banerjee SB, Gutzeit VA, Baman J, Aoued HS, Doshi NK, Liu RC, Ressler KJ. 2017. Perineuronal nets in the adult sensory cortex are necessary for fear learning. Neuron 95: 169-179.e3.

Bizley JK, Cohen YE. 2013. The what, where and how of auditory-object perception. Nat Rev Neurosci 14: 693-707.

Bozon B, Davis S, Laroche S. 2003. A requirement for the immediate early gene zif268 in reconsolidation of recognition memory after retrieval. Neuron 40: 695-701.

Carpenter-Hyland EP, Plummer TK, Vazdarjanova A, Blake DT. 2010. Arc expression and neuroplasticity in primary auditory cortex during initial learning are inversely related to neural activity. Proc Natl Acad Sci 107: 14828-14832.

Chawla MK, Lin G, Olson K, Vazdarjanova A, Burke SN, McNaughton BL, Worley PF, Guzowski JF, Roysam B, Barnes CA. 2004. 3D-catFISH: a system for automated quantitative three-dimensional compartmental analysis of temporal gene transcription activity imaged by fluorescence in situ hybridization. J Neurosci Methods 139: 13-24.

Cohen L, Rothschild G, Mizrahi A. 2011. Multisensory integration of natural odors and sounds in the auditory cortex. Neuron 72: 357-369.

Cruikshank SJ, Rose HJ, Metherate R. 2002. Auditory thalamocortical synaptic transmission in vitro. J Neurophysiol 87: 361-384.

Das B, Butler JS, Sherman F. 2003. Degradation of normal mRNA in the nucleus of Saccharomyces cerevisiae. Mol Cell Biol 23: 5502-5515.

DiCarlo JJ, Zoccolan D, Rust NC. 2012. How does the brain solve visual object recognition? Neuron 73: 415-434.

Dudai Y. 2012. The restless engram: consolidations never end. Annu Rev Neurosci 35: 227-247.

Ehret G. 1992. Categorical perception of mouse-pup ultrasounds in the temporal domain. Anim Behav 43: 409-416.

Ehret G, Haack B. 1981. Categorical perception of mouse pup ultrasound by lactating females. Naturwissenschaften 68: 208-209.

Ehret G, Haack B. 1982. Ultrasound recognition in house mice: key-stimulus configuration and recognition mechanism. J Comp Physiol [A] 148: $245-251$.

Ehret G, Koch M. 1989. Ultrasound-induced parental. Behavior in house mice is controlled by female sex-hormones and parental experience. Ethology 80: 81-93.

Ehret G, Koch M, Haack B, Markl H. 1987. Sex and parental experience determine the onset of an instinctive behavior in mice. Naturwissenschaften 74: 47.

Fletcher BR, Calhoun ME, Rapp PR, Shapiro ML. 2006. Fornix lesions decouple the induction of hippocampal arc transcription from behavior but not plasticity. J Neurosci 26: 1507-1515.

Fogarty MJ, Hammond LA, Kanjhan R, Bellingham MC, Noakes PG. 2013. A method for the three-dimensional reconstruction of Neurobiotin-filled neurons and the location of their synaptic inputs. Front Neural Circuits 7: 153.

Galindo-Leon EE, Lin FG, Liu RC. 2009. Inhibitory plasticity in a lateral band improves cortical detection of natural vocalizations. Neuron 62: 705-716. 
Gao M, Sossa K, Song L, Errington L, Cummings L, Hwang H, Kuhl D, Worley P, Lee HK. 2010. A specific requirement of Arc/Arg3.1 for visual experience-induced homeostatic synaptic plasticity in mouse primary visual cortex. J Neurosci 30: 7168-7178.

Grimsley JMS, Monaghan JJM, Wenstrup JJ. 2011. Development of social vocalizations in mice. PLoS One 6: e17460.

Gu Q. 2002. Neuromodulatory transmitter systems in the cortex and their role in cortical plasticity. Neuroscience 111: 815-835.

Guzowski JF, McNaughton BL, Barnes CA, Worley PF. 1999. Environment-specific expression of the immediate-early gene Arc in hippocampal neuronal ensembles. Nat Neurosci 2: 1120-1124.

Guzowski JF, Lyford GL, Stevenson GD, Houston FP, McGaugh JL, Worley PF, Barnes CA. 2000. Inhibition of activity-dependent arc protein expression in the rat hippocampus impairs the maintenance of long-term potentiation and the consolidation of long-term memory. $J$ Neurosci 20: 3993-4001.

Guzowski JF, Setlow B, Wagner EK, McGaugh JL. 2001. Experience-dependent gene expression in the rat hippocampus after spatial learning: a comparison of the immediate-early genes Arc, c-fos, and zif268. J Neurosci 21: 5089-5098.

Ivanova T, Matthews A, Gross C, Mappus RC, Gollnick C, Swanson A, Bassell GJ, Liu RC. 2011. Arc/Arg3.1 mRNA expression reveals a sub-cellular trace of prior sound exposure in adult primary auditory cortex. Neuroscience 181: 117-126.

Johnson SA, Cubberley G, Bentley DL. 2009. Cotranscriptional recruitment of the mRNA export factor Yra1 by direct interaction with the $3^{\prime}$ end processing factor Pcf11. Mol Cell 33: 215-226.

Kelly MP, Deadwyler SA. 2002. Acquisition of a novel behavior induces higher levels of Arc mRNA than does overtrained performance. Neuroscience 110: 617-626.

Kelly MP, Deadwyler SA. 2003. Experience-dependent regulation of the immediate-early gene arc differs across brain regions. J Neurosci 23: 6443-6451.

Khodadad A, Adelson PD, Lifshitz J, Thomas TC. 2015. The time course of activity-regulated cytoskeletal (ARC) gene and protein expression in the whisker-barrel circuit using two paradigms of whisker stimulation. Behav Brain Res 284: 249-256.

Korb E, Wilkinson CL, Delgado RN, Lovero KL, Finkbeiner S. 2013. Arc in the nucleus regulates PML-dependent GluA1 transcription and homeostatic plasticity. Nat Neurosci 16: $874-883$.

Lee HJ, Haberman RP, Roquet RF, Monfils MH. 2015. Extinction and retrieval+extinction of conditioned fear differentially activate medial prefrontal cortex and amygdala in rats. Front Behav Neurosci 9: 369.

Lin FG, Galindo-Leon EE, Ivanova TN, Mappus RC, Liu RC. 2013. A role for maternal physiological state in preserving auditory cortical plasticity for salient infant calls. Neuroscience 247: 102-116.

Liu RC, Schreiner CE. 2007. Auditory cortical detection and discrimination correlates with communicative significance. PLoS Biol 5: e173.

Liu RC, Miller KD, Merzenich MM, Schreiner CE. 2003. Acoustic variability and distinguishability among mouse ultrasound vocalizations. J Acoust Soc Am 114: 3412-3422.

Marlin BJ, Mitre M, D'Amour JA, Chao MV, Froemke RC. 2015. Oxytocin enables maternal behaviour by balancing cortical inhibition. Nature 520: $499-504$.

Messaoudi E, Kanhema T, Soulé J, Tiron A, Dagyte G, da Silva B, Bramham CR. 2007. Sustained Arc/Arg3.1 synthesis controls long-term potentiation consolidation through regulation of local actin polymerization in the dentate gyrus in vivo. J Neurosci 27: 10445-10455.

Miyashita T, Kubik S, Haghighi N, Steward O, Guzowski JF. 2009. Rapid activation of plasticity-associated gene transcription in hippocampal neurons provides a mechanism for encoding of one-trial experience. $J$ Neurosci 29: 898-906.

Morin JP, Quiroz C, Mendoza-Viveros L, Ramirez-Amaya V, Bermudez-Rattoni F. 2011. Familiar taste induces higher dendritic levels of activity-regulated cytoskeleton-associated protein in the insular cortex than a novel one. Learn Mem 18: 610-616.

Morin JP, Guzman-Ramos K, Bermudez-Rattoni F. 2015. New insights on retrieval-induced and ongoing memory consolidation: lessons from arc. Neural Plast 2015: 184083.
Muddashetty RS, Kelic S, Gross C, Xu M, Bassell GJ. 2007. Dysregulated metabotropic glutamate receptor-dependent translation of AMPA receptor and postsynaptic density-95 mRNAs at synapses in a mouse model of fragile X syndrome. J Neurosci 27: 5338-5348.

Nakayama D, Iwata H, Teshirogi C, Ikegaya Y, Matsuki N, Nomura H. 2015. Long-delayed expression of the immediate early gene Arc/Arg3.1 refines neuronal circuits to perpetuate fear memory. J Neurosci 35: 819-830.

Okuno H, Akashi K, Ishii Y, Yagishita-Kyo N, Suzuki K, Nonaka M, Kawashima T, Fujii H, Takemoto-Kimura S, Abe M, et al. 2012. Inverse synaptic tagging of inactive synapses via dynamic interaction of Arc/ Arg3.1 with CaMKIIß. Cell 149: 886-898.

Paxinos G, Franklin KBJ. 2001. The mouse brain in stereotaxic coordinates, 2nd ed. Academic, San Diego.

Ramirez-Amaya V, Vazdarjanova A, Mikhael D, Rosi S, Worley PF, Barnes CA. 2005. Spatial exploration-induced arc mRNA and protein expression: evidence for selective, network-specific reactivation. J Neurosci 25: $1761-1768$.

Ramirez-Amaya V, Angulo-Perkins A, Chawla MK, Barnes CA, Rosi S. 2013. Sustained transcription of the immediate early gene Arc in the dentate gyrus after spatial exploration. J Neurosci 33: 1631-1639.

Rolls ET. 2016. Pattern separation, completion, and categorisation in the hippocampus and neocortex. Neurobiol Learn Mem 129: 4-28.

Saha RN, Wissink EM, Bailey ER, Zhao M, Fargo DC, Hwang JY, Daigle KR, Fenn JD, Adelman K, Dudek SM. 2011. Rapid activity-induced transcription of Arc and other IEGs relies on poised RNA polymerase II. Nat Neurosci 14: 848-856.

Sheng M, McFadden G, Greenberg ME. 1990. Membrane depolarization and calcium induce c-fos transcription via phosphorylation of transcription factor CREB. Neuron 4: 571-582.

Shepard KN, Lin FG, Zhao CL, Chong KK, Liu RC. 2015. Behavioral relevance helps untangle natural vocal categories in a specific subset of core auditory cortical pyramidal neurons. J Neurosci 35: 2636-2645.

Shepherd JD, Bear MF. 2011. New views of Arc, a master regulator of synaptic plasticity. J Neurosci 14: 279-284.

Tagawa Y, Kanold PO, Majdan M, Shatz CJ. 2005. Multiple periods of functional ocular dominance plasticity in mouse visual cortex. Nat Neurosci 8: $380-388$.

Vazdarjanova A, Guzowski JF. 2004. Differences in hippocampal neuronal population responses to modifications of an environmental context: evidence for distinct, yet complementary, functions of CA3 and CA1 ensembles. J Neurosci 24: 6489-6496.

Vazdarjanova A, McNaughton BL, Barnes CA, Worley PF, Guzowski JF. 2002. Experience-dependent coincident expression of the effector immediate-early genes arc and Homer 1a in hippocampal and neocortical neuronal networks. J Neurosci 22: 10067-10071.

Wang KH, Majewska A, Schummers J, Farley B, Hu C, Sur M, Tonegawa S 2006. In vivo two-photon imaging reveals a role of arc in enhancing orientation specificity in visual cortex. Cell 126: $389-402$.

Waung MW, Pfeiffer BE, Nosyreva ED, Ronesi JA, Huber KM. 2008. Rapid translation of Arc/Arg3.1 selectively mediates mGluR-dependent LTD through persistent increases in AMPAR endocytosis rate. Neuron 59: 84-97.

Winer JA, Miller LM, Lee CC, Schreiner CE. 2005. Auditory thalamocortical transformation: structure and function. Trends Neurosci 28: 255-263.

Yasuda M, Fukuchi M, Tabuchi A, Kawahara M, Tsuneki H, Azuma Y, Chiba Y, Tsuda M. 2007. Robust stimulation of TrkB induces delayed increases in BDNF and Arc mRNA expressions in cultured rat cortical neurons via distinct mechanisms. I Neurochem 103: 626-636.

Zelikowsky M, Hersman S, Chawla MK, Barnes CA, Fanselow MS. 2014. Neuronal ensembles in amygdala, hippocampus, and prefrontal cortex track differential components of contextual fear. J Neurosci 34: 8462-8466.

Received June 23, 2017; accepted in revised form September 1, 2017. 


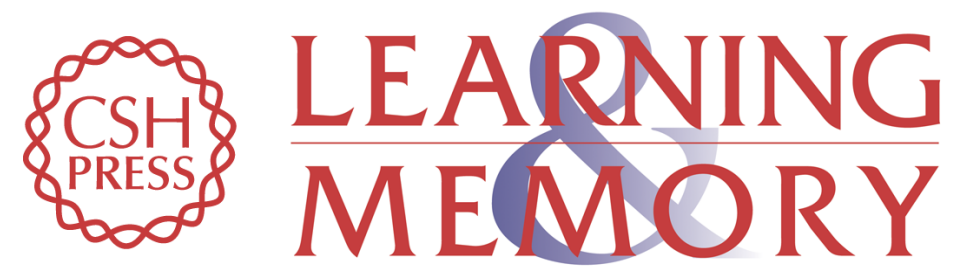

\section{Familiarity with a vocal category biases the compartmental expression of Arc/Arg3.1 in core auditory cortex}

Tamara N. Ivanova, Christina Gross, Rudolph C. Mappus, et al.

Learn. Mem. 2017, 24:

Access the most recent version at doi:10.1101/Im.046086.117

\begin{aligned} & \hline References $\begin{array}{l}\text { This article cites } 59 \text { articles, } 18 \text { of which can be accessed free at: } \\ \text { http://learnmem.cshlp.org/content/24/12/612.full.html\#ref-list-1 }\end{array} \\ & \begin{array}{r}\text { Creative } \\ \text { Commons } \\ \text { License }\end{array} \begin{array}{l}\text { This article is distributed exclusively by Cold Spring Harbor Laboratory Press for the } \\ \text { first } 12 \text { months after the full-issue publication date (see } \\ \text { http://learnmem.cshlp.org/site/misc/terms.xhtml). After } 12 \text { months, it is available under } \\ \text { a Creative Commons License (Attribution-NonCommercial } 4.0 \text { International), as } \\ \text { described at http://creativecommons.org/licenses/by-nc/4.0/. }\end{array} \\ & \begin{array}{c}\text { Receive free email alerts when new articles cite this article - sign up in the box at the } \\ \text { top right corner of the article or click here. }\end{array} \\ & \begin{array}{c}\text { Service } \\ \text { terting }\end{array}\end{aligned}$ 